\section{PROBABLE NATURE OF THE INTERNAL SYMMETRY OF CRYSTALS}

$S O M E$ studies pursued by the writer as to the nature of molecules have led him to believe that in the atom-groupings which modern chemistry reveals to th the several atoms occupy distinct portions of space and do not lose their individuality. The object of the present paper is to show how far this conclusion is in harmony with, and indeed to some extent explains, the symmetrical forms of crystals, and the argument may therefore in some sort be considered an extension of the argument for a condition of internal symmetry derived from the phenomena of cleavage.

If we are to suppose that crystals are built up of minute masses of different elements symmetrically disposed, it is natural t) inquire in the first place what very symmetrical arrangements of points or particles in space are possible.

It would appear that there are but five, which will now be described.

If a number of equal cubes are built into a continuous mass (Fig. I), a system of points occupying the centres and angles of these cubes will furnish an example of one of these symmetrical arrangements. In this system each point is equidistant from the eight nearest points, and if a number of equal-sized spheres be stacked on a base layer arranged so that the sphere centres when joined form a system of equal squares, a side of which bears to the diameter of the spheres the ratio $2: \sqrt{3}$ (see plan a), the sphere centres in such a stack will also furnish an example of this first kind of symmetry (Fig. 2).

A second ki id of symmetry will be presented if one-half the points in the first kind be removed so that we have only those at the cube centres, or only those at the cube angles. In this system each point is equidistant from the six nearest points, and if equal-sized spheres be stacked upon a base layer, arranged so that the sphere centres when joined form a system of equilateral triangles, a side of which bears to the diameter of the spheres the ratio $\sqrt{2}:$ I (see plan $b$ ); and if the layers be so placed that the sphere centres of the fourth layer are over those of the first, those of the fifth over those of the second, and so on, the sphere centres in such a stack will also furnish an example of this second kind of symmetry ( $F$ ig. 3).

A third kind of symmetry will be presented if again one-half the points be removed, i.e. so that when cubes of two colours arranged in such a way that each cube is surrounded by cubes of the other colour are used (see Fig. I), we have only the points at the centres of the cubes of one colour. In this system each point is equidistant from the twelve nearest points, and if equalsized spheres be stacked upon a base layer in which the spheres are in contact, and whether they form a square pattern (see plan $c$ ), or a triangular one (see plan $d$ )-previded that, if triangular-pattern layers be employed, the sphere centres in the fourth layer must be over those in the first, those in the fifth over those in the second, and so on-the sphere centres (the arrangement being the same in either case) will furnish a second example of the third kind of symmetry (Figs. 4 and $4 a$, the latter showing a stack with the angle removed to display the triangular arrangement).

A fourth kind of symmetry, which resembles the third in that each point is equidistant from the twelve nearest points, but which is of a widely different character from the three former kinds, is depicted if layers of spheres in contact arranged in the triangular pattern (plan $d$ ) are so placed that the sphere centres of the third layer are over those of the first, those of the fourth over those of the second, and so on. The symmetry produced is hexagonal in structure and uniaxal (Figs. 5 and $5 a$ ).

A fifth kind of symmetry, and this completes the number of very symmetrical arrangements possible, resembles the second kind of symmetry in that each point is equidistant from the six nearest points, and bears the same relation to the fourth kind (Fig. 5) as the second (Fig. 3) bears to the third (Fig. 4); that is to say, it may be regarded as produced by the insertion of additional points in positions midway between points arranged in the fourth kind of symmetry. It is depicted if triangularly constituted layers identical with those depicting the second kind of symmetry (plan $b$ ) are deposited in the following way (Fig. 6) -First place three layers as though to produce the second kind of symmetry; then place the fourth with its sphere centres over those of the second layer; then the fifth so that the third, fourth, and fifth, like the first, second, and third, are in the second kind of symmetry; then the sixth with its sphere centres over those of the fourth and second ; and then the seventh, so that the fifth, sixth, and seventh layers are also in the second kind of sym. metry; and so on. The symmetry produced is, like the last, hexagonal in structure and uniaxal.

The writer believes that every one of the various symmetrical forms presented by crystals can be shown to be consistent with the subsistence of an arrangement of the atoms of the crystallising compound in one or other of these five kinds of symmetry at the

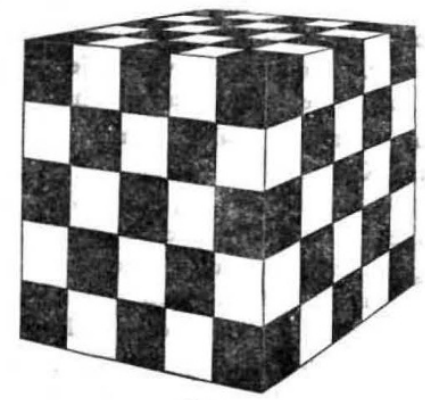

FIG. I.

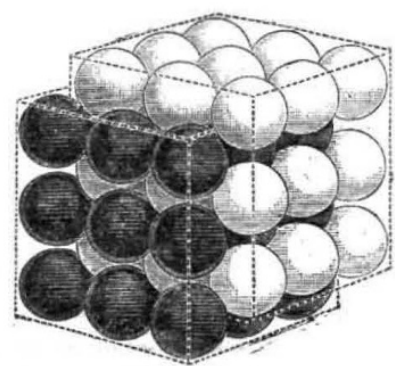

FIG. 2.

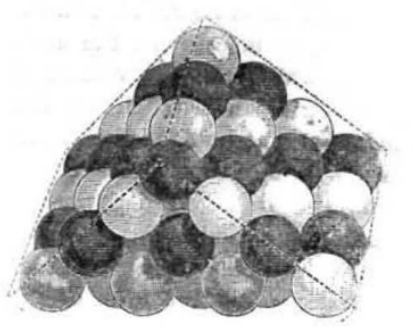

Fig. 3.

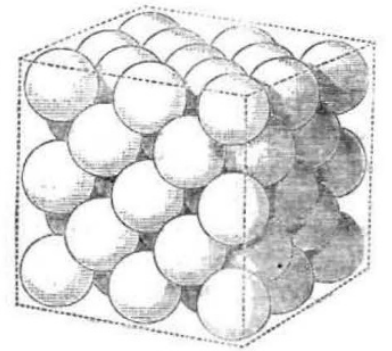

FIG. 4 .

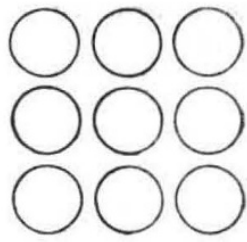

Plan $a$.

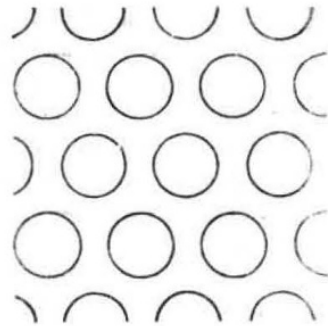

Plan b.

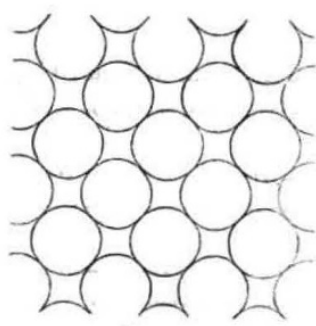

Plan $c$. time when crystallisation begins; and proposes to show that a relation subsists between the atomic composition of very many bodies and their crystal forms in harmony with this conclusion.

To proceed then to facts, we notice first that, as a rule, compounds consisting of an equal number of atoms of two kinds crystallise in cubes.

The following may be mentioned:-

Potassic chloride, $\mathrm{KCl}$.

Potassic bromide, $\mathrm{KBr}$ (sometimes elongated into prisms, or extended into planes). 
Potassic iodide, $\mathrm{KI}$.

Sodic chloride, $\mathrm{NaCl}$.

Sodic bromide, $\mathrm{NaBr}$.

Sodic iodide, $\mathrm{NaI}$ (anhydrous above $40^{\circ} \mathrm{C}$.).

Casi : chloride, $\mathrm{CS} . \mathrm{Cl}$.

Plumbic sulphide, $\mathrm{PbS}$.

Argentic chloride, $\mathrm{AgCl}$

When we have named lithic chloride, crystallising above $15^{\circ}$

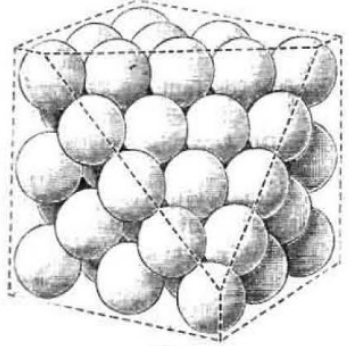

Fig. $4 a$.

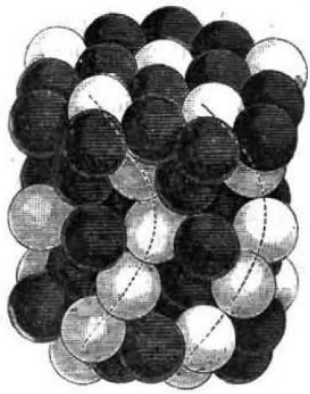

FIG. 5.

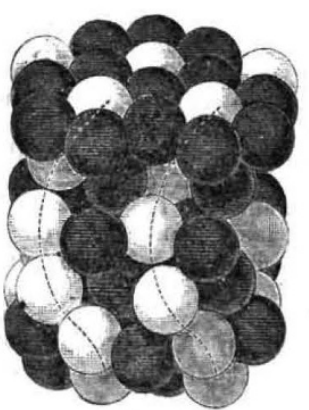

F1G. $5 a$.

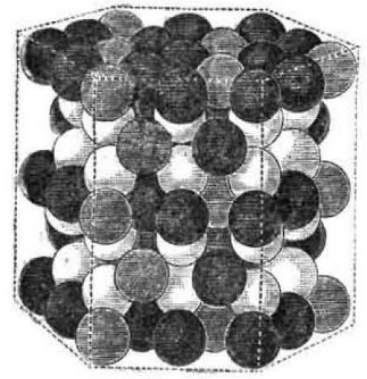

Fig, 6.

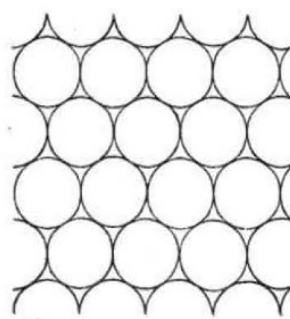

Plan $d$.
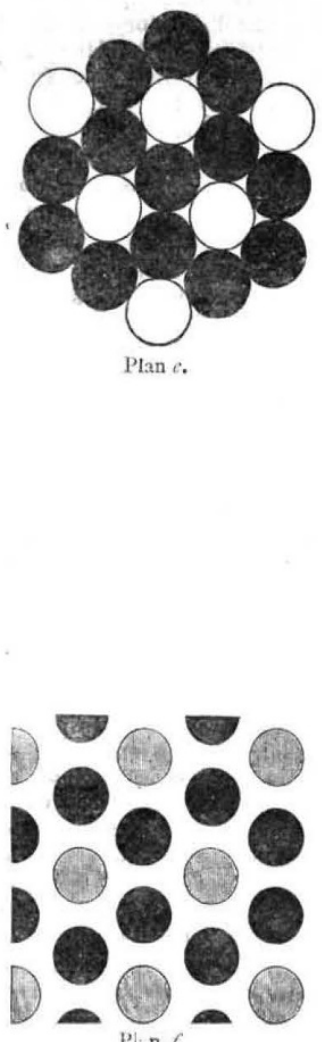

in octahedra, we have mentioned most of the compounds consisting of two elements in equal proportions known to us in a crystalline state.

Mercuric sulphide, $\mathrm{Hg} . \mathrm{S}$, which crystallises in six-sided prisms, is an apparent exception, but if we were guided by the gaseous volume of mercury in determining its atomic weight, we should have to write the compound $\mathrm{Hg}_{2} \mathrm{~S}$.
Other apparent exceptions are :-

Zincic oxide, $\mathrm{ZnO}$, crystallising in six-sided prisms.

Cadmium sulphide, Cds; and

Glucina, GO, crystallising in minute six-sided prismatic crystals.

Now three out of our five possible kinds of internal symmetry have three axes or directions at right angles to each other, in reference to which they are disposed in the same symmetrical manner, and two kinds, the first and second, admit of a very symmetrical arrangement of two kinds of particles in equal numbers (see Figs, 2 and 3). Surely this coincidence is very significant, and at lea t suggests the probability that when a compound consists of two kinds of chemical atoms in equal num. bers, these atoms are symmetrically placed according to either the first or the second kind of internal symmetry.

We observe next that the third and fourth kinds of symmetry (Figs. 4 and 5) readily lend themselves to the symmetrical arrangement of particles of two kinds present in the proportion I : 2. For, as already pointed out, these two kinds of sym. metry may either of them be produced by piling up layers of spheres placed triangularly in contact (see plan d), and spheres of two colours present in the proportion of $2:$ I can be arranged in a most symmetrical manner in layers of this kind (see plan $e$ ).

As to what varieties of position of bi-coloured layers of this kind with respect to one another are possible, consistent with great symmetry, we have concluded that, apart from the ques. tion of arrangement of colour, there are but two, viz. the third and fourth kinds of symmetry (Figs. 4 and 5); but taking colour into account a greater variety is possible. Thus a little con. sideration shows us tbat, while all the possible ways of depositing the second layer produce a practically identical result, a choice of six different equally symmetrical results is presented in depositing the third layer, in all of which the spheres of the less numerous colour form files of sphere; in contact running through the layers, and three of wbich belong to the third kind of symmetry and three to the fourth.

To specify these : We may have the less numerous spheres of the third layer placed with respect to those in the second and first :--

(1) So that the three spheres of each of the files just above alluded to range in line, the lines joining their centres forming a series of parallel straight lines crossing the planes of the layers obliquely. This arrangement belongs to the third kind of symmetry.

( 2 and 3 ) So that the centres of these three spheres, when joined, form a slightly obtuse angle; a different result being produced as the angle is made to the right or to the left. This pair of arrangements belongs also to the third kind of symmetry.

(4) So that the less numerous spheres in the third layer are vertically over those in the first. This arrangement belongs to the fourth kind of symmetry.

(5 and 6) So that, $a_{i}$ in (2) and (3), the tri llets of spheres form a system of equal obture angles, but the angles now being very obtuse. There are here, as in (2) and (3), a right-handed and a left-handed arrangement. These belong to the fourth kind of symmetry.

The deposition of the third layer, by the necessities of symmetry, determines the deposition of succeeding layers, and it follows therefore from the above that six different equally symmetrical arrangements of spheres of two colours present in the proportion $2: 1$ are possible in the third and fourth kinds of symmetry.

As to (I) the parallel files of the less numerous spheres crossing the first three layers will extend through subsequent layers.

As to (2) and (3) every three continuou; layers will display the less numeruu; sphere centres placed to form the same angles as are presented by the triplets in the first three layers, and consequently these sphere centres lie on spirals which are right-handed or left-handed as the case may b : ; the less numerous spheres in the fourth layer bein $r$ vertically over those in the first, those in the fifth over those in the seco.d, and sj on.

As to (4) the less numerous spheres in the fourth layer must lie vertically over those in the second, those in the fifth over those in the third, and so on; and thu; the files of spheres in contact running through successive layers form a series of similar zigzags.

As to (5) and (6) the sphere centres, as in (2) and (3), lie either on right-handed or on left-handed sjirals; in this cas s the less numerous spheres in the seventh layer boing vertically 
over those in the first, those in the eighth over those in the second, and so on (Figs. 5 and $5 a$ ).

When we inquire whether the symmetrical arrangements just traced are in harmony with the facts respecting compounds of two kinds of atoms in the proportions $\mathbf{I}: \mathbf{2}$, we find some very important evidence.

Thus water, $\mathrm{H}_{2} \mathrm{O}$, crystallises in six-sided prisms or in rhombohedra; forms both of which are compatible with one or other of the above symmetrical arrangements.

And the following most interesting concurrence of facts indicates that the symmetrical arrangements in the fourth kind of symmetry above described (see Figs. 5 and $5 a$ ) are those of the atoms of quartz.

(a) Quartz consists of oxygen two atoms, silicon one atom; just the proportions in these arrangements.

(b) It has the property of circular polarisation, from which it has been proved that its molecules must have a spiral arrangement, and, since some crystals have the property of rotating in one direction, some in the opposite, that this spiral arrangement is rigbt-handed in some crystals, left-handed in others.

(c) It crystallises in six-sided prisms terminated by six-sided pyramids, a form derivable, as we have seen, from the arrangements before us.

As to this last point, just a word of explanation why we must not look for the angles exhibited by our model arrangements to be identical with the angles made by the pyramid faces in quartz.

It is a matter of common observation that the process of crystallisation is generally associated with change of bulk, and if we suppose this change to arise from expansion, or contraction, generally expansion of the different kinds of atoms, and that these different kinds have different degrees of expansion, we see that a mass symmetrically arranged in the manner supposed will in crystallising expand or contract more in some directions tban in others, and while we should look for a similar change in the direction of each of the thrte transverse subordinate axes of the crystal, we should look for a different change in the direction of the principal axis. And thus, supposing the mass when liquid immediately before it began to crystallise to have had the internal symmetry which has been depicted, it is evident that the untqual chan e of dimension in different directions might suffice to bring about such an inclination of the faces of the terminal pyramids to the sides of the prism as is actually found to exist.

In support of this explanation we have the fact that crystals not of the regular system have been found to expand unequally in different directions when subjected to heat.

Further evidence in support of the theories here submitted is found in the fact that, with scarcely any exception, the compounds we are now considering do not crystallise in the regular or cubic system. ${ }^{1}$ WILliam BarLOW

\section{(To be continued.)}

\section{THE HELVETIC SOCIETY OF NATURAL SCIENCES}

THE sixty-sixth session of this Society was held early in the month of August of the present year in the city of Zurich. The proceedings if the various Mathematical, Pbysical, Chemical, Zoological, Botanical, and Medical Departments are somewhat fully reported in the Archives des Sciences Physiques et Nuturelles, Geneva, October 15. On August 6 a preliminary meeting was held of the delegates of the (antonal Sections and Special Comn ittees, and next day the ses:ion was formally opened in the Town Hall under the presidency of Prof. Cramer. The two ensuing days were devoted to the work of the several Sections, all of which were well at tended by numerous Swiss and foreign savants, brought together by the double attraction of the Helvetic Society and the National Exhibition, which was also held this year in Zurich.

In the Mathematical Section, over which Prof. W. Fiedler presided, the chief papers were those of Prof. Geiser (Zurich), on surfaces of the third degree; of Dr. Rudio (Zurich), on the geodetic lines traced on surfaces of the second degree ; of Prof. Fiedler (Zuricb), on the inter ection of equilateral hyperboloids revolving on parallel axes.

In the Physical Section, presided over by Prof. R. Clausius,

' With regard to calcic fluoride (fuor-spar), which appears as an exception it may be remarked that a different atumic weight for calcium which would enable us to u rite the compound $\mathrm{CaF}$ would enable us to get over a difficult with regard to another compound of calcium, as we shall see presently.
M. F. A. Forel (Morges) communicated the result of his researches made to determıne the lımits of variation of temperature in the waters of Lake Geneva. According to his thermometrical soundings, the diurnal variation is perceptule down to a depth of from 1o to 15 metres; the summer variation from 60 to 100 metres. Exceptional winters like that of $1879-80$ are felt as low as 334 metres. Since that year the temperature of the water at these great depths has been raised on an average about half a degree Centigrade.

Some preliminary results of his researches on the refraction and dispersion of crystallised alums were communicated by $M$. Charles Soret of Geneva. By means of his completely reflecting refractometer, described in the Archives for January, 1883 , the author has determined the indices of refraction for the principal lines of the solar spectrum from $a$ to $\mathrm{G}$ inclusively for six sulphuric alums with alumina base.

Prof. Clausius read a pafer of practical importance on the theory and proper method of construction of dynamo-electric machines. Some curious exferiments were made by M. C. de Candolle of Geneva, showing how ripples are formed on sandy surfaces at the bottom of the sea. From these experiments it results that the phenomenon is produced by the friction of a hquid mass agaiust any substance more viscous than itself. Hence the sand may ke regarded as forming with the water a viscous mixture, on the surface of which the friction of the pure liquid develops ripples in the same way that the friction of the air develops ripples on the surface of the water itself.

Amongst the other nemuirs in the Physical Section the most notew orthy were those of Prof. H. F. W eber (Zurich) on liquids and gases as heat coldductors ; an experimental demonstration of the second principle of the mechanical theory of heat, by $M$. Racul Pictet ; on the determination of the ohm, by Prof. H. F. Weber; on the results of the observations and researches made in the laboratory of the Lausanne Academy on atmospheric electricity, by M. Henri Dufour of Lausanne. The author described several successful attempts made by him to reprcduce artificially the elecuic phenomena observed in the terrestrial atmosphere.

The Chemical Section was opened, under the presidency of Prof. Wislicenus, ty Yrof. V. Meyer's memoir on the nature of the chemical elements according to recent research. The author leaus to the vieus of Mendeleeff and Lothar Meyer, who regard the properties of simple bodies as the pericdical functions of their atomic weights. The fact that Mendeléeff was able to predict the existence of galiium and scandium, ard correctly determine their atomic weights, was adduced in support of the theory that all the elements are merely different compound forms or one primitive substance. Hence, although bitherto baffled, the attempts now being made to decompose them may result in the exptrimental determination of one absolute primordial substance.

Prof. F. Krafft (Basle) presented some higher alcohols of the series $\mathrm{C}_{\mathrm{n}} \mathrm{H}_{2 \mathrm{n}}+2 \mathrm{O}$, accompanying them with some rewarks on the synthesis of alcohols in gereral. A résumé was given by Prof. Louis Soret (Geneva) of his researches un the ab: orption of the ultra-violet rays by various substances of animal origin. The author dwelt on the great importat ce of this branch of spectral analysis to chemistry, and concluded with a brief description or the method and appliances used by him in his original re: earches,

Uther valuable chemical papers were those of Dr. M. Cérésole (Lausanne), on acetacetic acids; of Prot. V. Meyer on the apparatus used in determining the densities of gas es at very high temperatures; of Yrof. Schulze, describing the researches nade by bim jointly with M. J. Barbieri on phenylamidopropionic acid, $u$ lich is obtained by heating albuminoid substances with chlorhydric acid and chloride of tin; of Prof. Wislicenus (Wurtzbury), on the relation of the optical rotatory power of carburets of hydrogen, on the existence of an aton: of asymmetric carbon, and on the procucts of the reaction of dichloride of phtalyle on the sodic comtination of malonic ether; Prof, G. Lunge (Zurich), on the formation of sulphuric acid in lead chambers; Lr. Urech (Stuttgart), on a lamp fed Ly ether of petroleum. This laml', constructed by C. C. Lilienfein, of Stuttgart, consists of a metallıc receiver containing the ether (f petroleum, and connected with a Bunsen burner slightly modified in consequence of the liquid nature of the combustible.

In the Zoological zection Prof. C. Vogt, president, the proceedings were opened by a communication from Prof. $\mathrm{H}$. Fol (Geneva), on the physiological origin of the individual in the 